$\xi=-1$

\title{
A novel approach for phishing emails real time classification using k-means algorithm
}

\author{
Vidya Mhaske-Dhamdhere ${ }^{1} *$, Sandeep Vanjale ${ }^{2}$ \\ ${ }^{1}$ P. h D. Research scholar, Bharati Vidyapeeth Deemed University College of Engineering, Pune \\ ${ }^{2}$ Professor Bharati Vidyapeeth Deemed University College of Engineering, Pune \\ *Corresponding author E-mail: Vidya.dhamdhere@gmail.com
}

\begin{abstract}
The dangers phishing becomes considerably bigger problem in online networking, for example, Facebook, twitter and Google+. The phishing is normally completed by email mocking or texting and it frequently guides client to enter points of interest at a phony sites whose look and feel are practically indistinguishable to the honest to goodness. Non-technical user resists learning of anti-phishing technic. Also not permanently remember phishing learning. Software solutions such as authentication and security warnings are still depending on end user action.

In this paper we are mainly focus on a novel approach of real time phishing email classification using K-means algorithm. For this we uses 160 emails of last year computer engineering students. we get True positive of legitimate and phishing as $67 \%$ and $80 \%$ and true negative is $30 \%$ and $20 \%$., which is very high so we ask same users reasons which I mainly categories into three categories ,look and feel of email, email technical parameters, and email structure.
\end{abstract}

Keywords: Email and Websites Phishing; Phishing Detection Techniques; User Awareness on Email Phishing.

\section{Introduction}

Users might reach to phishing sites through some social networking sites like Facebook, Twitter. Attackers typically target specific cluster of indivuals organizations to get intellectual information, business secrets or military data rather than gain. This variation of general phishing is called Spear phishing. Whaling may be a kind of spear phishing where target of group may be a larger fish like military offices personal business and government agencies.antiphishing techniques like blacklist, whitelist, heuristic and visual similarity primarily based approaches became less effective in detecting work phishing websites. The limitation of blacklisting is that phishing sites that are not listed in blacklist don't seem to be detected. These kind of non-backlisted phishing sites are referred to as Zero day phishing sites.

\section{Related work}

Alejandro, Eduardo [2] authors uses neural framework approach. to get to the two techniques utilizes RF (Random Forest) and LSTM (a long/here and now memory mastermind on datasets phish tank and Common Crawl, which gives result as precision rate of $93.5 \%$ and $98.7 \%$.RF and LSTM utilizes 14 highlights of lexical and quantifiable examination of url resembles space exist in Alexa rank, subdomain length, URL length, way length, URL Entropy, '@'and '- ' character tally in URL.

Anndita, Dhirendra [1] utilizes gathering learning approach has been utilized for phishing email identification. The model incorporates of three stages preprocessing, highlight inspecting, characterization arrange. Add up to 97 messages utilized out of that 96 effectively order and one misclassify. Encourage forward neural system to group tried email into phish or ham email in light of separated email header and body. Distinguishing proof rate is 98\%. Author has considered ID rate, acknowledgment rate, redress rate, misclassification rate or error rate, precision of characterization.

Ankit Kumar Jain B.B. Gupta [3] these author has design a novel approach to protect against phishing attacks at client side. $75 \%$ of phishing web sites used for 5 top level domains specifically .com, .tk, .pw, .cf, net. webpages usually contains a login page and when a user opens the fake webpage and inputs personal information .online users wont able to differentiate between phishing and confide webpages.one of the effective solutions to a phishing attack is to integrate security measures with the net browser which may raise the alters whenever a phishing web site is accessed by an phisher. a novel approach categorized in to 4 steps.

1) Create phishing web sites.

2) Writes associate email and includes the link of phishing web sites and send to authorized users.

3) The user opens the email and visits the phishing websites. The phishing web sites ask the user to input personal information.

4) After getting users personal information used for money or another advantages.

Experimental results show that $86 \%$ correct positive rate and $48 \%$ false negative rate. Analysis is done on three parameters namely no link, null hyperlinks, and quantitative relation o hyperlinks pointing to a main domain.

Hassan Y. A, Abdelfettah Belghith [4] these author has implemented Case Based Reasoning Phishing Detection System(CBR_PDS), which three stages, which are Lure, Hook and Catch. The Lure is all around created email that looks true and authority.it will guide to client to phony site. The Hook is phony site that copy real site in which client can uncover his qualifica- 
tions. The Catch incorporates the utilization of delicate data gathered by deceitful activity. For this 572 phishing email datasets is used. This CBR_PDS framework give precision 95.62\%.main drawback is that phishing sites have a short lifecycle, which means a classifier should be trained frequently to keep track of almost phishing websites in order to enhance the accuracy.

Marjan Abdeyadan, Rayat Pisheh [5] has design internet phishing attacks detection life cycle including three phases: early stage,mid phishing stage, post phishing stage.in the beginning times, the phisher gets ready for phishing and makes an email or a spam and send it to the clients. In the mid phishing stages, the casualties get the phony messages and uncover their touchy and significant data.in the third cycle of phishing, taking data is conferred. The high rate of web use among phone clients has made numerous business and money related administrations be given through the web.

Data robbery of phishing is security challenge which is normally done by sending spam messages and emails.in the dataset utilized by the writers, honest to goodness, suspicious, more, phishing addresses are appeared by estimations of $01,0,-1$ separately. for identifying phishing sites uses features like age of source site, nearness of IP address in the connection, linguistic mistake, the nearness of @ character in the connection on FP,FN,TN,TP of proposed strategy are $99.62 \%, 032 \%, 0.5 \%, 99.5 \%$ and $99.7 \%$.the precision of preparing information archives $94 \%$.

Melad Mohamed, Nurlinda Basir, Madihah Mohd Saudi [6] authors has preparing strategies ought to be intended to pull in clients consideration keeping in mind in the end goal to upgrade their mindfulness and influence them to hold gained learning for longer time. Preparing exercises accordingly, must consider information obtaining, Information maintenance and information exchange aspects. Phishers are generally target hostile to phishing frame works through ignorance and mindlessness elements of internet users. Anti-phishing preparing material can be conveyed to learners through many channels, for example, messages, publication, classrooms and amusements.

According to information security Forum (ISF),"security mindfulness is a procedure of learning by which, student understand the significance of data security issues, the security level required by the association and people's security duties. Three key segments of security level mindfulness, they are, continuous or consistent process, learning conveyance techniques and people's conduct impact. The advantage of inserted preparing over other conventional preparing strategies is that, it can learning into other related fields. Posted articles and tips about phishing is another type of internet preparing strategies such materials and frequently published by government's and different associations and groups for example ,Federal Trade Commission and Anti-phishing Working Group.anti-phishing Phil demonstrates how web based amusements can enable clients to recognize phishing sites by showing them where to search for phishing signs in web programs.it additionally demonstrate to clients generally accepted methods to accurately land to honest to goodness locales through web indexes. Amusement architect have detailed that False Positive (FP) limited to $14 \%$ from $30 \%$ and False Negative (FN) rate likewise limited to $17 \%$ from $34 \%$.

Mouna Jouinia,Latifa Ben,Arfa Rabaia,Anis Ben[7] has proposed a security risk grouping model, which enables us to think about the dangers class affect rather than a risk affect as a risk differs.

1) Mutually restrictive-every danger should fit in at most one class.

2) Exhaustive-All danger examples

3) Unambiguous-all classes must be clear and exact with goal.

4) Repeatable-results in similar characterization

5) Accepted-all classification are sensible.

6) Useful-It can be utilized to pick up knowledge into the field of request.

The criteria order list got from the outline are:

1) Security danger source: the beginning of risk either interior or outside.
2) Security danger operations-the specialists that reason dangers and we recognized three primary classes: Human, natural .Mechanical.

3) Security risk inspiration-the objective of aggressors on a framework which can be noxious or non-malevolent security risk expectation.

The model recognized the danger impacts: Destruction of data, corruption of data, Theft/loss of data, Disclosure of data, foreswearing of utilization, Elevation of benefit and illegal use.74.3\% of the misfortunes are cause by infections, unapproved access, tablet or versatile equipment robbery and burglary of exclusive data. $70 \%$ of extortion is executed by insiders instead of by outside. $90 \%$ of security controls are centered on outer threats.

Narenda Shekolkar, chaitali Shahetc.[8] has used Link Guard algorithm for phishing detection. Link Guard works by breaking down the contrasts between the visual connection and the real link.it first concentrates the DNS names from the genuine and the visual connection .it at that point looks at the real and visual DNS names, if these names are not the same, at that point it is phishing of class.

Nayeem Khan, Johari Abdullah, Adnan Shahid Khan [9] these author has design methodologies for defending malicious script attacks using machine learning classifies algorithm Naïve Bayes. Security is based on to correlative methodologies, signature based and heuristic based identification approaches. The signature based approach depends on the identification of one of a kind string designs in the paired code. Heuristic based recognition depends on the arrangement of master choice guidelines to identify the attacks.it will just recognize adjusted or variation existing malware. The drawback of utilizing this approach is that it takes a long time in performing checking and examination, which radically backs off the security execution. Another issue of the approach is that it presents numerous false positive. False positive happens when a framework wrongly recognizes code or a record as malignant when really it is not.

Naive Bayes classifier consider precision, preparing time, linearity, the quantity of parameters, number of highlights are used. 70 highlights of JavaScript's as appeared in the Reference section. The proposed approach accomplished a precision of $100 \%$ in recognition for already obscure malevolent JavaScript based on learning. Exploratory outcomes demonstrate that ROC-1 was accomplished by KNN classifies with no false positive. The wrapper technique assumed an essential part in highlight determination, which prompts high precision contrasted with other examined static methodologies.

Ratinder Kaur and Maninder Singh[10]has proposed novel hybrid framework that coordinates inconsistency for identifying and breaking down zero day attaks.the framework is actualized and assessed against different standard measurements True Positive Rate(TPR),False Positive Date(FPR), F- Measure, Total Accura$\mathrm{cy}(\mathrm{ACC})$ and Receiver Operating Characteristic(ROC).the outcome indicates high discovery rate with almost zero false positive.to guard against zero day attacks, the exploration group has proposed different procedures. There are partitioned into Statistical based, Signatured based, behavior based and Hybrid strategies. Anupama Aggarwaly, Ashwin Rajadesingan,[11]has present PhishAri expansion works for chrome program is composed in JavaScript. PhishAri use $\mathrm{d}$ for detection phishing real time on Twitter. Twitter Streaming API 12 and the Channel work given API to gather such Tweets. The API takes the tweets ID as info and returns back a string showing weather the tweet is phishing or safe. Phishers have a tendency to have a great deal of @ tags in their tweets with the goal that their tweet is straightforward.

Detecting phishing via web based networking is test as results

1) Vast volume of information-online networking enables clients to effortlessly share their values of information,

2) Constrained space- Twitters 140 character restriction the substance due to which clients utilizes shorthand documentations.

3) Quick change-web based networking changes quickly making phishing location troublesome. 
4) Shorten URL's- phishing URLs are abbreviated to the objective URL.

It is hard to distinguish phishing on Twitter dissimilar to messages on account of the fast spread of phishing joins in the system, short size of the substance, utilization of URL confusion.twwets substance and its attributes like length, hash tags, mentions the Twitter client posting the tweet for example age of the record, number of tweets and the supporter follower ration. Random forest classifiers works best to phishing tweet reorganization on dataset with high precision of $92.52 \%$.

Routhu Srinivasa, Syed Taqi Ali [12] has design heuristic approach of phishshield.It takes input as address and output the standing of address a phishing or legitimate website. The heuristic use to observe phishing area unit footer links with null price, zero links in body of HTML,copyright content ,title content and website identity.to develop tool PhishSheild, author used Net Beans 8.02,IDE,JAVA complier, Jsoup ,API and firebug tool. Jsoup is used for parsing the HTML contents of webpages and extracting HTML content like links in footer, copyright, title, CS. firebug open supply Firefox extension that is employed for debugging, editing and monitoring of nay website's CSS, HTML, Dom, XHR and JavaScript. the main advantage of Phishsheild application is that it will observe phishing sites that tricks the users by substitution content with images, that most of the prevailing anti phishing techniques not capable to observe, though they will take lot of execution time the accuracy rate obtained for phishsheild is $96 \%$. Abdulghani Ali Ahmed, Nurul Amirah Abdullah [13] these author has implemented real time phishing detection of websites Using Term Frequency -Inverse Archive Frequency (TF_IDF).the phisher makes a shadow site that appears to be like the genuine site. Users regularly have numerous client accounts on different sites including social system, email and furthermore represent banking.

The phishing sites by utilizing TF-IDF system recover data and content mining effectively diminishes the false positive rate. Total 97 phishing webpage with around $6 \%$ false positive rate.prevenion strategies for site mocking are survived and ordered into different methodologies: content based, heuristic based and boycott based approaches. This approach utilizes a mix of stateless page assessment, sate full page assessment and examination of archive post information to register proxy file system.

Boycott based approach is recovering the URLs from phishing pages with a specific end goal to keep up and make the blacklist. the security danger of the web pages with a specific end goal is highlight of criteria ,for example, time of internet uses, create web server review, no. of time visiting site page. Nation that facilities the site, name of association that facilitating the present site and hazard rating. Some highlights can be numerous, for example,URLs ,area ,personality, security and encryption, source code, page style and substance, web address bar and social human factor.

This examination concentrates just on URLs and area name highlights .highlights of URL and space names are checked utilizing a few criteria ,for example ,IP address, long URL address, includeing a prefix or addition, diverting utilizing the images, use of double slash and URL having the image of @.

Qian Cui[14]has design novel tracking phishing attacks using clustering algorithm.in this approach undertake to intrinsic characteristics of phishing sites, such as the presence of specific sort of internet forms, or some unusual structures in URLs. $90 \%$ of the attacks are repeats of previous attacks. Also $90 \%$ of the actual attacks in list can mechanically remove. There are 18 cluster active for one month and in general average period of time of cluster is 25 days. Attack instance s will be clustered in such the simplest way that every one of the instances of a similar attack in the same cluster, associate degree attack category, showing few variations of the Dom, and lot of variations in terms of domain names and ultimately scientific discipline addresses of the machine serving the attacks.

A content based methodology victimization a Term Frequency and Inverse Document Frequency (TF-IDF) analysis to spot the phishing target. The keyword extracted by the TF-IDF algorithmic rule on a given pages are submitted to look engines like Google and output the possible tag get of phishing attacks with $99 \%$ true positive.

S. Carolin Jeeval, Elijah Blessing Rajsingh [15] has present phishing URL detection using apriori association rule mining algorithm. The proposed techniques compromise of two stages.

1) URL LOOK and feel stage.

2) Highlight extraction phase.

It was discovered that $77.75 \%$ of phished URLs are with uncommon characters, $9.4 \%$ o phished URLs contained IP address, $64 \%$ of phished URL are observed as subdomain used, $66.5 \%$ of phishing URL are found without top level domain.apriori give $99 \%$ exactness level.

\section{Methodology}

According to [16-17] for user phishing awareness training is essential. User awareness training can be do following 4 ways.

1) Articles

2) presentation

3) Audio and video

4) Quiz

In [16-17] paper author has use presentation method and Quiz method. Quizzes are used for testing user's knowledge about phishing email and websites in first training approach. in second training approach presentation is used, thorough with shows phishing emails and legitimate emails and explain why particular email is phishing or legitimate. For that use real time emails received by author on his email id. Even with this training do's and don't also explain.to identify phishing or legitimate emails visualization, technical parameter and email header and body, these three categories are used which is shown in below table.

Table 1: Different Factors in Determine Decisions about Email Legitimate and Phishing Emails

\begin{tabular}{|c|c|c|c|c|}
\hline Judgment criteria & & Phishing & Legitimate & Unable to identify \\
\hline \multirow{4}{*}{ Visualization (Look and Feel) } & Different Colures used in emails & Present in email & & \multirow{11}{*}{$\begin{array}{l}\text { Present in email } \\
\text { Present in email }\end{array}$} \\
\hline & Plain text email & & Present in email & \\
\hline & Org. logo or trademarks in email signature & & Present in email & \\
\hline & Copy right of email signature & & Present in email & \\
\hline \multirow{7}{*}{$\begin{array}{l}\text { Technical parameters used in } \\
\text { email }\end{array}$} & There is https in URL & & Present in email & \\
\hline & There is no https in URL & & Present in email & \\
\hline & Email is embedded URL or link & & Present in email & \\
\hline & Email is no embedded URL or link & & & \\
\hline & Verification process of data & & & \\
\hline & Manually URL checking & Present in email & & \\
\hline & Sender email address is unknown & Present in email & & \\
\hline \multirow{5}{*}{ Email header and body } & Personalized email & Present in email & & \multirow{5}{*}{ Present in email } \\
\hline & Other personal data & & & \\
\hline & Typing mistake /grammatical error & Present in email & & \\
\hline & Promoting offers/opportunities & Present in email & & \\
\hline & Use of urgent or forceful language & Present in email & & \\
\hline
\end{tabular}




\subsection{Experiment}

For this training total 16 emails are shown to 179 users, which is shown in below table. Out of 16 emails only 5 emails are legitimate and 11 are phishing with users identification result is shown in table 2.

\subsection{Experiment results}

In training $67 \%$ users correctly identify legitimate email and $80 \%$ phishing emails are identified.

If we compare before and after training approach only $28 \%$ users legitimate email correctly identification is improvement and 39\% phishing email identification improvement, which is very less so that we required to solve this problem machine learning algorithms are required.

After training we take review of users why they incorrectly classify legitimate email as phishing and phishing email as legitimate. They give reason like multicolor are used in email, email embedded URL is given, sender is unknown, email signature is not proper, domain and subdomain is not register. According to reason given by participant which is shown in table 3 according to each emails are summaries.

Table 2: Training Email Classification Done by Users

\begin{tabular}{|c|c|c|c|c|c|c|c|c|c|c|c|c|c|c|c|c|}
\hline 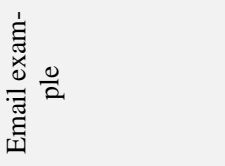 & 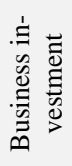 & 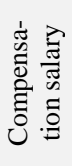 & 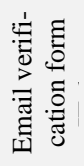 & 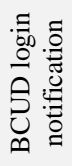 & 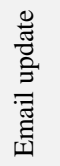 & 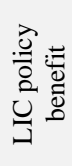 & 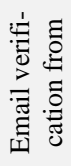 &  &  &  & 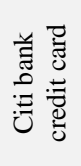 & 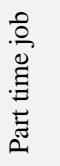 & 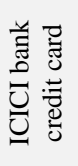 & 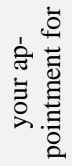 & 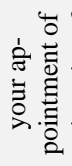 & 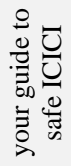 \\
\hline Legitimate & 7 & 52 & 58 & 129 & 52 & 148 & 24 & 27 & 14 & 62 & 41 & 40 & 37 & 110 & 146 & 149 \\
\hline Phishing & 172 & 123 & 112 & 44 & 123 & 22 & 148 & 150 & 161 & 114 & 129 & 136 & 135 & 62 & 28 & 28 \\
\hline Unable to identify & 0 & 4 & 9 & 6 & 4 & 9 & 7 & 2 & 4 & 3 & 9 & 3 & 7 & 7 & 5 & 2 \\
\hline
\end{tabular}

Table3:

\begin{tabular}{|c|c|c|c|c|c|}
\hline \\
\hline Sr.no & Email title & $\begin{array}{l}\text { Email is phishing } \\
\text { or legitimate }\end{array}$ & $\begin{array}{l}\text { Count of Cor- } \\
\text { rectly classify }\end{array}$ & $\begin{array}{l}\text { Count of incor- } \\
\text { rectly classify }\end{array}$ & $\begin{array}{l}\text { Correctly classify as legitimate or phishing Reason } \\
\text { given by participants }\end{array}$ \\
\hline 2 & $\begin{array}{l}\text { Compensation salary in- } \\
\text { crease }\end{array}$ & Phishing & 172 & 52 & $\begin{array}{l}\text { 1. Email header name is not finance company name or } \\
\text { bank name. } \\
\text { 2.for more information click here link is given } \\
\text { 3. email signature and header is mismatch } \\
\text { 4. For contact no email is and contact number is giv- } \\
\text { en. } \\
\text { 5. Email is colorful. } \\
\text { 1. Domain name is not register domain. } \\
\text { 2. Email start is informally. } \\
\text { 3. For conformation link is given. Details are not } \\
\text { given in mail. } \\
\text { 4. Forcing user to do not share salary increase details } \\
\text { to anyone. } \\
\text { 5. Email sender is unknown. }\end{array}$ \\
\hline 3 & $\begin{array}{l}\text { Email verification form IT } \\
\text { dept. }\end{array}$ & Phishing & 112 & 58 & $\begin{array}{ll}\text { 1. } & \text { University never contact to student direct- } \\
\text { ly. } & \\
2 . & \text { Domain is not register domain. } \\
\text { 3. } & \text { College email id is not verified form uni- } \\
\text { versity. } & \end{array}$ \\
\hline 4 & BCUD login notification & Legitimate & 129 & 44 & $\begin{array}{l}\text { 1. Email start is informal. } \\
\text { 2. For query contact number and email id is given. } \\
\text { 3. Sender is known. } \\
\text { 4. For updating of BCUD user and password link is } \\
\text { not given. }\end{array}$ \\
\hline 5 & Email update & & 123 & 52 & $\begin{array}{l}\text { 1.Email sender is unknown } \\
\text { 2. Email signature is doubtful. } \\
\text { 3. Asking user to configure your email to outlook web } \\
\text { access. }\end{array}$ \\
\hline & & Legitimate & & & $\begin{array}{l}\text { 1. LIC benefit mandate from, cancel cheque, } \\
\text { NEFT details asking. }\end{array}$ \\
\hline 6 & LIC policy benefit & Legitimate & 148 & 22 & $\begin{array}{l}\text { 2. } \\
\text { query. } \\
\text { 3. }\end{array}$ \\
\hline 7 & $\begin{array}{l}\text { Email verification from } \\
\text { university }\end{array}$ & Phishing & 148 & 24 & $\begin{array}{ll}\text { 1. } & \text { Domain name is not register domain. } \\
2 . & \text { Informally email started. } \\
\text { 3. } & \text { Email signature is missing. } \\
\text { 4. } & \text { Email embedded link Is given. }\end{array}$ \\
\hline 8 & $\begin{array}{l}\text { Important email from uni- } \\
\text { versity }\end{array}$ & Phishing & 150 & 27 & $\begin{array}{l}\text { 1. University never contact to staff and student direct- } \\
\text { ly. } \\
\text { 2. Email embedded link is given. } \\
\text { 3. Email header and signature is mismatch. }\end{array}$ \\
\hline 9 & $\begin{array}{l}\text { Deposit fund from universi- } \\
\text { ty }\end{array}$ & Phishing & 161 & 14 & $\begin{array}{l}\text { 1. In email lastly I do not take call is written. } \\
\text { 2. Domain is not register domain. } \\
\text { 3. Sender the unknown. }\end{array}$ \\
\hline 10 & $\begin{array}{l}\text { Bank transfer alter from } \\
\text { Citi bank }\end{array}$ & Phishing & 114 & 62 & $\begin{array}{ll}\text { 1. } & \text { Asking user to open attachment of file. } \\
2 . & \text { Sender is unknown. } \\
3 . & \text { Email signature is informal. }\end{array}$ \\
\hline
\end{tabular}




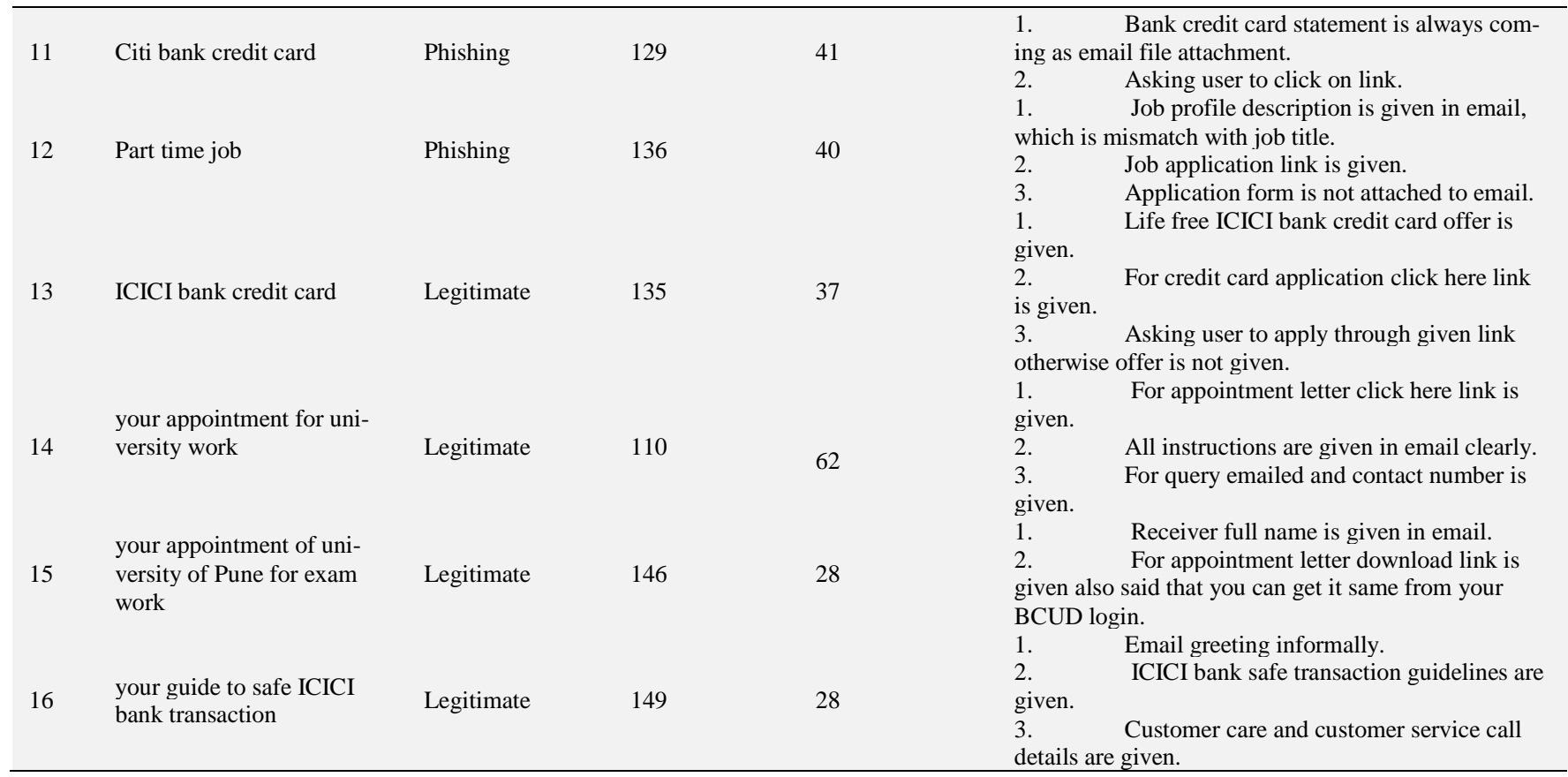

\section{Conclusion}

User awareness about email and websites phishing is one of the necessary aspects. Existing literature survey user education was done on-line or offline. User education ought to provide ceaselessly. In existing user, 18 to twenty 25 years, gender, and country, that wasn't spare parameter analysis the performance of user.to find this analysis gap we have a tendency to area unit progressing to embrace additional parameter like age within the completely different range, education, profession, daily work net usages.

If we have a tendency to compare before and once coaching approach $28 \%$ users legitimate email properly identification is improvement and $39 \%$ phishing email identification improvement, that is extremely less so that we have a tendency to needed to resolve this downside machine learning algorithms area unit required

\section{References}

[1] Anandita, Dhirendra Pratap Yadav, Priyanka Paliwal, Divya Kumar, Rajesh Tripathi," A Novel Ensemble Based Identification of Phishing E-Mails", Conference ICMLC 2-17, February 24-26, 2017, Singapore, Singapore. 2017 ACM.

[2] Alejandro Correa Bahnseny, Eduardo Contreras Bohorquez, Sergio Villegas.” Classifying Phishing URLs Using Recurrent Neural Networks", 978-1-5386-2701-3/17/\$31.00 c 2017 IEEE.

[3] Ankit Kumar Jain and B. B. Gupta," A novel approach to protect against phishing attacks at client side using auto-updated whitelist", EURASIP Journal on Information Security (2016) 2016 https://doi.org/10.1186/s13635-016-0034-3.

[4] Hassan Y. A. Abutair, Abdelfettah Belghith," Using Case-Based Reasoning for Phishing Detection ", the 8th International Conference on Ambient Systems, Networks and Technologies ANT2017, Procedia Computer Science 109C (2017) 281-28 Published by Elsevier B.V.

[5] Marjan Abdeyazdan1, and Ali Rayat Pisheh2," Detecting internet phishing attacks using data mining methods", 3 rd International conference on Innovative Engineering Technologies (ICIET'2016) August 5-6, 2016 Bangkok (Thailand)

[6] Melad Mohamed Al-Daeef, Nurlida Basir, Madihah Mohd Saudi,' Security Awareness Training: A Review", Proceedings of the World Congress on Engineering 2017 Vol I WCE 2017, July 5-7, 2017, London, U.K.

[7] Mouna Jouinia ,Latifa Ben Arfa RabaiaAnis Ben Aissa," Classification of security threats in information systems", 5th International Conference on Ambient Systems, Networks and Technologies (ANT-2014), 2014 Elsevier.
[8] Narendra. M. Shekokar, Chaitali Shah, Mrunal Mahajan,Shruti Rachh," An Ideal Approach For Detection And Prevention Of Phishing Attacks", Elsevier, Procedia Computer Science 49 ( 2015 ) 82 - 91. https://doi.org/10.1016/i.procs.2015.04.230.

[9] Nayeem Khan, Johari Abdullah, and Adnan Shahid Khan," Defending Malicious Script Attacks Using Machine Learning Classifiers", Hindawi Wireless Communications and Mobile ComputingVolume 2017, Article ID 5360472, doi.org/10.1155/2017/5360472.

[10] Ratinder Kaur and Maninder Singh," A Hybrid Real-time Zero-day Attack Detection and Analysis System", I. J. Computer Network and Information Security, 2015, 9, 19-31 Published Online August 2015 in MECS (http://www.mecs-press.org/)

[11] Routhu Srinivasa Rao* and Syed Taqi Ali," PhishShield: A Desktop Application to Detect Phishing Webpages through Heuristic Approach", Eleventh International Multi-Conference on Information Processing-2015 (IMCIP-2015) 1877-0509 (C) 2015 The Authors. Published by Elsevier.

[12] Anupama Aggarwaly, Ashwin Rajadesingan_, Ponnurangam Kumaraguru," PhishAri: Automatic Realtime Phishing Detection on Twitter", IEEE-2012.

[13] ABDULGHANI ALI AHMED, NURUL AMIRAH ABDULLAH," Real Time Detection of Phishing Websites", 978-1-5090-09961/16/\$31.00 @2016 IEEE.

[14] Qian Cui," Tracking Phishing Attacks over Time", 2017 International World Wide Web Conference Committee (IW3C2 April 3-7, 2017, Perth, Australia. 978-1-4503-4913-0/17/04 https://doi.org/10.1145/3038912.3052654.

[15] S. Carolin Jeeva1* and Elijah Blessing Rajsingh2," Intelligent phishing url detection using association rule mining", Hum. Cent. Comput. Inf. Sci. (2016) 6:10 Spinger open acess@ 2016.

[16] Vidya Mhaske Dhamdhere,Prasanna Joeg," To Study of phishing attacks and user behavior", IEEE, $2^{\mathrm{ND}}$ INTERNATIONAL CONFERENCE ON INVENTIVE COMPUTATION TECHNOLOGIES,2017.

[17] Vidya Mhaske Dhamdhere,Dr. Sandeep Vanjale,Dr. Prassana Joeg," To study user behavior using phishging education",International Conference on Applied Sciences,enginerring,technology and management(ICASETM-17), Nov.2017. 